JOURNAL OF THE

AMERICAN MATHEMATICAL SOCIETY

Volume 17, Number 2, Pages 373-388

S 0894-0347(03)00447-8

Article electronically published on December 2, 2003

\title{
ISOPERIMETRIC INEQUALITIES IN CRYSTALLOGRAPHY
}

\author{
ANTONIO ROS
}

\section{INTRODUCTION}

The study of the isoperimetric problem in the presence of crystallographic symmetries is an interesting unsolved question in classical differential geometry: Given a space group $\mathcal{G}$, we want to describe, among surfaces dividing Euclidean 3-space into two $\mathcal{G}$-invariant regions with prescribed volume fractions, those which have the least area per unit cell of the group. We know that this periodic isoperimetric problem always has solutions and any of these solutions is a smooth triply periodic surface with constant mean curvature $([3,8,12,20,21])$. The explicit description of these surfaces, which we call $\mathcal{G}$-isoperimetric surfaces, remains open in most of the cases. Let us consider, for instance, the following two simple situations. Assume the prefixed volume fractions are (both) equal to $1 / 2$. If $\mathcal{G}$ is the cubic integer lattice $\mathbb{Z}^{3}$, then it was proved by Hadwiger [10] that the $\mathcal{G}$-isoperimetric surface is, up to symmetries, the family of parallel planes $x=n / 2, n \in \mathbb{Z}$. If $\mathcal{G}$ is the group of symmetries of $\mathbb{Z}^{3}$ (Pm $\overline{3} m$ in crystallographic notation), then it is conjectured that the isoperimetric surface is the classical $P$ Schwarz triply periodic minimal surface. From the point of view of applications, it is also important to obtain explicit isoperimetric inequalities for periodic regions with prescribed symmetries.

Triply periodic minimal surfaces and related surfaces are widely accepted in crystallography and materials science; see for instance the papers [6, 13, 17, 29, 30. They appear as interface models in mesoscale self-assembled phenomena. Some of these are reminiscent of the familiar soap bubbles (such as lipid-water systems), but others are of a different nature (such as diblock copolymers). Roughly speaking, these interfaces are periodic and separate two immiscible liquid materials. The size of the periods varies between tens and hundreds of nanometers and the more interesting cases usually present cubic symmetry. Mesoscale interfaces can be treated as periodic surfaces minimizing (under volume fraction constraints) a suitable energy, whose dominant term is, in several cases, the area of the interface itself. So the periodic isoperimetric problem is the simplest geometric model to explain the shapes of these interfaces. Among the surfaces appearing most frequently in this context are periodic arrays of planes, spheres and cylinders, and small perturbations of constant mean curvature $P(\mathcal{G}=P m \overline{3} m)$ and $D(\mathcal{G}=F d \overline{3} m)$ Schwarz surfaces and the $G$ Schoen Gyroid $\left(\mathcal{G}=I 4_{1} 32\right)$.

Received by the editors March 17, 2003.

2000 Mathematics Subject Classification. Primary 53A10, 53C42, $20 \mathrm{H} 15$.

Key words and phrases. Isoperimetric problem, periodic minimal surfaces, cubic symmetry.

Partially supported by MCYT-FEDER research projects BFM2001-3318. 
In this paper we will consider the periodic isoperimetric problem in the case where $\mathcal{G}$ is one of the 36 cubic space groups, viewed as a finite group acting on the unit cubic torus $T=\mathbb{R}^{3} / \mathbb{Z}^{3}$. A cubic group $\mathcal{G}$ does not leave invariant any direction in the space or, equivalently, $\mathcal{G}$ contains $\langle 111\rangle 3$-fold axes (i.e., 3 -fold axes parallel to the four body diagonals of the cube). For details about these groups see, for instance, the International Tables for Crystallography [11] and the Bilbao Crystallographic Server [18. For our purposes, the main invariants associated to a cubic group $\mathcal{G}$ will be $k(\mathcal{G})$, the minimum number of points in an orbit of $\mathcal{G}$, and $\chi(\mathcal{G})$, the largest Euler characteristic among nonspherical $\mathcal{G}$-isoperimetric surfaces. The first one can be checked directly, and in Theorem 8 and $\$ 4$ we will give upper estimates for the second one; see Table 1 In particular we will prove that we always have $\chi(\mathcal{G}) \leq-4$. Our main result is a sharp isoperimetric inequality for $\mathcal{G}$-invariant regions in terms of $k(\mathcal{G})$ and $\chi(\mathcal{G})$. Moreover, since this inequality is optimal for small volumes, we solve explicitly the $\mathcal{G}$-isoperimetric problem in that range. In particular we will obtain the following result.

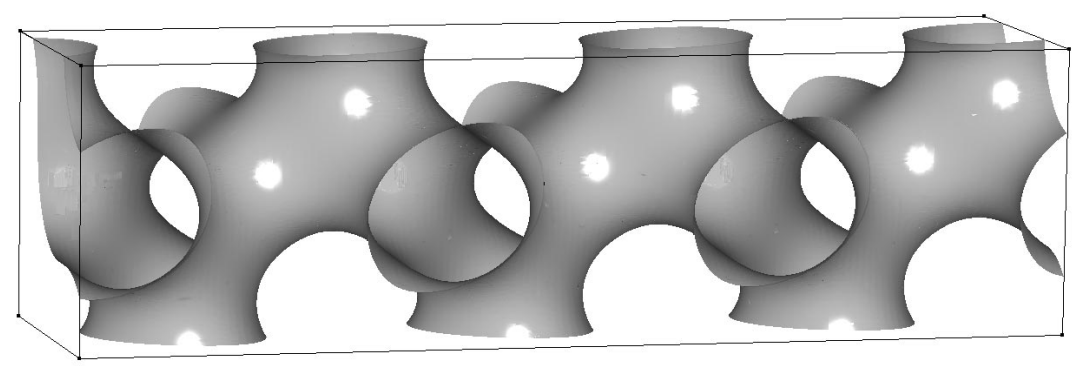

Figure 1. The minimal Gyroid $G$ was discovered by A. Schoen [28]. As a distintive symmetry, it has $4_{1}$-fold axes (i.e., screw axes of order four) parallel to the edges of the cube. It is conjectured that the Gyroid has least area among surfaces dividing the 3-space into two $I 4_{1} 32$-invariant regions with equal volume fractions.

Theorem 1. Let $\mathcal{G}$ be a cubic space group, $k=k(\mathcal{G})$ the minimum number of points in an orbit of $\mathcal{G}$ and $\chi=\chi(\mathcal{G})$ the largest Euler characteristic among nonspherical $\mathcal{G}$-isoperimetric surfaces in the cubic torus $T=\mathbb{R}^{3} / \mathbb{Z}^{3}$. Then any $\mathcal{G}$-isoperimetric surface enclosing a volume smaller than or equal to

$$
\frac{\chi^{2}}{2(4 k-\chi)(2 k-\chi)}
$$

is a (disjoint) union of $k$ spheres centered at the points of a smallest orbit of $\mathcal{G}$.

Any surface $\Sigma \subset T$ separating the torus into two $\mathcal{G}$-invariant regions with the same volumes satisfies

$$
A(\Sigma)>\left(\frac{9 k \pi(-\chi)(2 k-\chi)}{(4 k-\chi)^{2}}\right)^{1 / 3} .
$$

In Table 1 we have listed the values $k(\mathcal{G})$ and our estimates of $\chi(\mathcal{G})$ for all the cubic groups. Then we give the estimates of Theorem 1 particularized to each one of these groups. 
TABLE 1. The first column contains cubic space groups $G$ (acting on the torus $\left.T=\mathbb{R}^{3} / \mathbb{Z}^{3}\right)$. The second column gives the smallest number $k$ of points in a $\mathcal{G}$-orbit. The third column gives an upper bound on the Euler characteristic $\chi$ of $\mathcal{G}$-isoperimetric surfaces other than spheres. The fourth column gives a lower bound on $v_{s p h}$ ( $\mathcal{G}$-isoperimetric surfaces enclosing a volume $v<v_{s p h}$ are spherical). The last column gives a lower bound on the area of any surface dividing the cubic torus into two $\mathcal{G}$-invariant regions of equal volume.

\begin{tabular}{lrrrc}
\hline Cubic space groups & $k$ & $\chi \leq$ & $v_{\text {sph }}>$ & $A>$ \\
\hline$P 23, P 432, P m \overline{3}, P \overline{4} 3 m, P m \overline{3} m$ & 1 & -4 & $1 / 6$ & 2.19 \\
$P 4_{2} 32, P \overline{4} 3 n, P n \overline{3}, P n \overline{3} n, P m \overline{3} n, P n \overline{3} m$ & 2 & -4 & $1 / 12$ & 2.32 \\
$P 2_{1} 3, P 4_{1} 32\left(P 4_{3} 32\right), P a \overline{3}$ & 4 & -8 & $1 / 12$ & 2.92 \\
$I 23, I 432, I m \overline{3}, I \overline{4} 3 m, I m \overline{3} m$ & 2 & -8 & $1 / 6$ & 2.76 \\
$I 2_{1} 3, I 4_{1} 32$ & 8 & -8 & $1 / 30$ & 3.00 \\
$I a \overline{3}$ & 8 & -16 & $1 / 12$ & 3.69 \\
$I \overline{4} 3 d$ & 12 & -16 & $1 / 20$ & 3.75 \\
$I a \overline{3} d$ & 16 & -16 & $1 / 30$ & 3.78 \\
$F 23, F m \overline{3}, F \overline{4} 3 m$, & 4 & -16 & $1 / 6$ & 3.48 \\
$F 4_{1} 32, F d \overline{3}, F \overline{4} 3 c, F m \overline{3} c, F d \overline{3} m$ & 8 & -16 & $1 / 12$ & 3.69 \\
$F d \overline{3} c$ & 16 & -16 & $1 / 30$ & 3.78 \\
$F 432, F m \overline{3} m$ & 4 & -32 & $4 / 15$ & 3.97 \\
\hline
\end{tabular}

We can compare Table 1 with Table 2, which gives the area of several concrete periodic constant mean curvature surfaces (including some minimal surfaces) which are candidates for solving the isoperimetric problem with equal volume fractions for various groups $\mathcal{G}$. In some cases the exact value of the area is known, as in the first three rows (see Table 3), and in the other cases the value has been computed numerically; see [9, 17] and in particular 2]. Note that we give two surfaces with $F m \overline{3} m$ symmetry. The area of the first one is exact, but the value for the second one is only a crude approximation taken from the graphics of [2]. So is not yet clear which one is better.

The next theorem contains our general isoperimetric inequality. The volumearea profile of $k$ spheres, giving the area of $k$ spheres of the same radius in term of the enclosed volume, is $J_{k}(v)=\left(36 k \pi v^{2}\right)^{1 / 3}$.

Theorem 2. Let $\mathcal{G}$ be a cubic group, let $k=k(\mathcal{G})$ and let $\chi$ be a negative integer with $\chi \geq \chi(\mathcal{G})$. If $\Omega$ is a $\mathcal{G}$-invariant region in the torus $T=\mathbb{R}^{3} / \mathbb{Z}^{3}$ with volume $V$, then the area $A$ of the boundary surface $\partial \Omega$ satisfies $A \geq J_{k, \chi}(V)$, where $J_{k, \chi}$ : $[0,1] \rightarrow \mathbb{R}$ is the function defined as follows:

$$
\begin{gathered}
J_{k, \chi}(v)=J_{k}(v), \quad \text { for } \quad 0 \leq v \leq v_{0}=\frac{\chi^{2}}{2(4 k-\chi)(2 k-\chi)}, \\
J_{k, \chi}(v)=J_{k}(1-v), \quad \text { for } \quad 1-v_{0} \leq v \leq 1,
\end{gathered}
$$

and $J_{k, \chi}$ is the solution of the equation

$$
J^{2} J^{\prime \prime}+J\left(J^{\prime}\right)^{2}-4 \pi \chi=0, \quad \text { with } \quad J\left(v_{0}\right)=J_{k}\left(v_{0}\right) \quad \text { and } \quad J^{\prime}\left(v_{0}\right)=J_{k}^{\prime}\left(v_{0}\right),
$$

for $v_{0} \leq v \leq 1-v_{0}$. 
TABLE 2. $\Sigma$ is a constant mean curvature surface with group symmetry in the first column and dividing the 3 -space into two equal volume fractions. The surfaces in this column are natural candidates for solving the $\mathcal{G}$-isoperimetric problem. The columns $\chi(\Sigma), H$ and $A(\Sigma)$ give the Euler characteristic, the mean curvature and the approximate area (per cubic cell) of this surface, respectively.

\begin{tabular}{lcrrr}
\hline Space group & $\Sigma$ & $\chi(\Sigma)$ & $H$ & $A(\Sigma)$ \\
\hline$P m \overline{3} m$ & $P$ Schwarz & -4 & 0 & 2.35 \\
$F d \overline{3} m$ & $D$ Schwarz & -16 & 0 & 3.84 \\
$I 4{ }_{1} 32$ & $G$ Schoen & -8 & 0 & 3.09 \\
$I m \overline{3} m$ & double $P$ & -8 & $\neq 0$ & 4.05 \\
$I m \overline{3} m$ & $I$ - $W$ P Schoen & -12 & $\neq 0$ & 3.40 \\
$P n \overline{3} m$ & double $D$ & -4 & $\neq 0$ & 3.35 \\
$F m \overline{3} m$ & covering of $P(2 \mathrm{a})$ & -32 & 0 & 4.68 \\
$F m \overline{3} m$ & $F$ - $R D$ Schoen & -40 & $\neq 0$ & 4.70 \\
$I a \overline{3} d$ & double $G$ & -16 & $\neq 0$ & 5.49 \\
$I \overline{4} 3 d$ & $S$ Fischer \& Koch & -40 & 0 & 6.85 \\
$I a \overline{3} d$ & $\Gamma$ cylinders & 0 & $\neq 0$ & 6.59 \\
\hline
\end{tabular}

It will be clear after the proof of the theorem that $J_{k, \chi}(v)$ is symmetric with respect to $v=1 / 2$. In applications of the above isoperimetric inequality, it may be convenient to use the following explicit parameterization of the nonspherical part of $J_{k, \chi}$ : The graph of $J_{k, \chi}$ between $v_{0}$ and $1-v_{0}$ coincides with the trace of the curve

$$
\gamma_{k, \chi}(t)=\left(\frac{1}{2}+A_{0} t+\frac{2}{3} \pi \chi t^{3}, A_{0}+2 \pi \chi t^{2}\right)
$$

where

$$
A_{0}=\left(\frac{9 k \pi(-\chi)(2 k-\chi)}{(4 k-\chi)^{2}}\right)^{1 / 3} \quad \text { and } \quad|t| \leq\left(\frac{3 k^{2}}{\pi(-\chi)(4 k-\chi)(2 k-\chi)}\right)^{1 / 3} .
$$

The isoperimetric profile of a cubic group $\mathcal{G}$ is the function $I_{\mathcal{G}}:[0,1] \rightarrow \mathbb{R}$ defined by

$$
I_{\mathcal{G}}(v)=\min \{A(\partial \Omega) \mid \Omega \subset T \text { is a } \mathcal{G} \text { - invariant region with } V(\Omega)=v\},
$$

where $V(\Omega)$ is the volume of $\Omega$ and $A(\partial \Omega)$ is the area of its boundary. Theorem 2 can be stated as $I_{\mathcal{G}} \geq J_{k, \chi}$. In the Figures 2 , 3 and 4 we give $J_{k, \chi}$ for the groups $P m \overline{3} m, F d \overline{3} m$ and $I 4_{1} 32$ using the data in Table 1 In the three cases $J_{k, \chi}$ is the lower concave curve joining the points $(0,0)$ and $(1,0)$. The upper concave curve joining the same points corresponds to the volume-area of concrete $\mathcal{G}$-symmetric surfaces: spheres, for small and large volumes, and surfaces equidistant from the minimal $P$ and $D$ Schwarz surfaces and the $G$ Schoen Gyroid, respectively (the range where these equidistant surfaces are free of singularities is given by Lemma 3 and Table 3 ). Thus, the isoperimetric profile $I_{\mathcal{G}}$ lies between these two graphs. Note the accuracy of the isoperimetric inequality of Theorem 2 for these three groups (especially for the last one, $\mathcal{G}=I 4_{1} 32$ ). It seems natural to hope that the following assertion holds. 


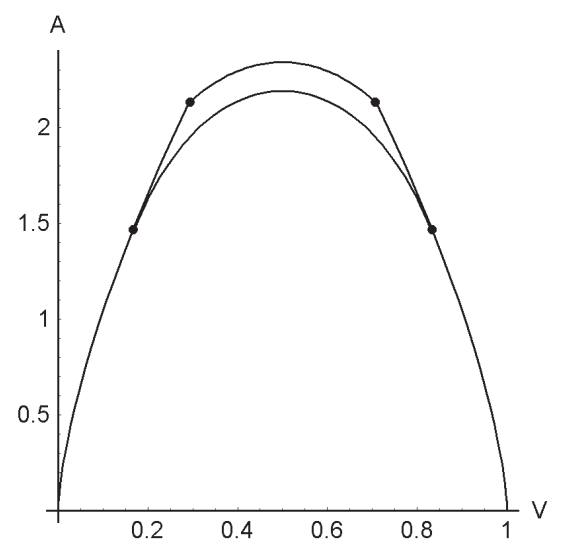

FiguRE 2. Upper and lower bounds of the isoperimetric profile for $P m \overline{3} m$-invariant regions. The upper curve consists of surfaces equidistant to the minimal Schwarz $P$ surface (central part between the two highest dots) and spheres (remaining volumes). The lower curve is the graph of $J_{1,-4}$ : two spherical parts joined by a concave arc between the two lowest dots.

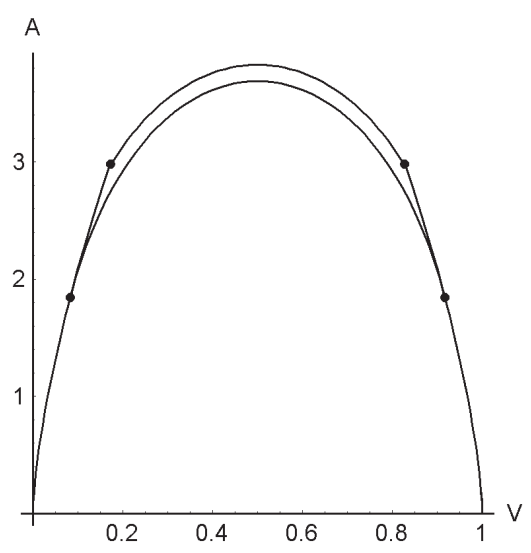

FiguRE 3. Upper and lower bounds of the isoperimetric profile for $F d \overline{3} m$-invariant regions: volume-area of spheres and equidistant surfaces to the $D$ surface (top curve) and $J_{8,-16}$ (bottom curve).

Conjecture 1. The isoperimetric surfaces for the groups $P m \overline{3} m, F d \overline{3} m$ and $I 4_{1} 32$ consist of spheres, for small and large volumes, and constant mean curvature $P, D$ and $G$ surfaces, for intermediate volumes, respectively. In particular, the minimal $P$ and $D$ Schwarz surfaces and the minimal $G$ Schoen Gyroid minimize the area among surfaces dividing the torus into two regions with the above symmetries and equal volumes.

The constant mean curvature $P, D$ and $G$ surfaces are surfaces obtained by deforming the minimal ones inside the family of surfaces with the same orientationpreserving symmetries. 


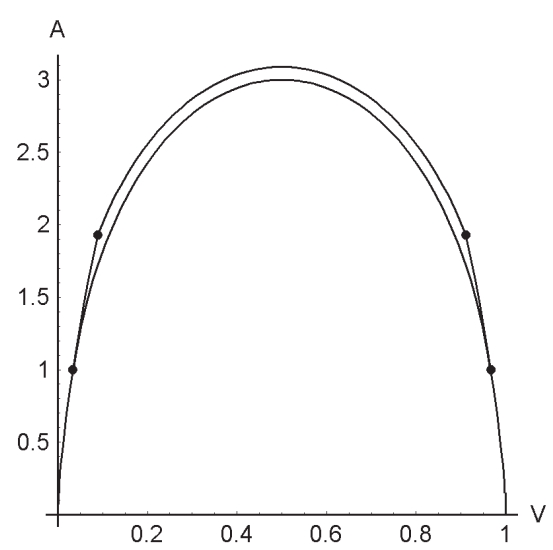

FiguRE 4. Upper and lower bounds of the isoperimetric profile for $I 4_{1} 32$-invariant regions: volume-area of spheres and equidistant surfaces to the Gyroid surface $G$ (top) and $J_{8,-8}$ (bottom).

Finally we mention another interesting question: to decide which cubic space groups have the same isoperimetric surfaces. We give below an (incomplete) list of families which seem to verify this property.

Conjecture 2. The groups in each one of the following families have the same isoperimetric regions:

(1) $P 23, P 432, P m \overline{3}, P \overline{4} 3 m, P m \overline{3} m$;

(2) $P 2_{1} 3, P 4_{1} 32$;

(3) $I 23, I 432, \operatorname{Im} \overline{3}, I \overline{4} 3 m, \operatorname{Im} \overline{3} m$;

(4) $I 22_{1} 3, I 4_{1} 32$;

(5) $F 432, F m \overline{3} m$.

In the case where $\mathcal{G}$ is one of the groups in the first row of Table 1, the results of Theorems 1 and 2 have been proved in [12].

\section{Preliminaries}

In this paper $\mathcal{G}$ will denote a cubic space group acting on the unit cubic 3 -torus $T=\mathbb{R}^{3} / \mathbb{Z}^{3}$. This means that $\mathcal{G}$ is a finite group of symmetries of $T$ with 3 -fold rotation axes along the $\langle 111\rangle$ directions (i.e., 3-fold axes are parallel to the four body diagonals of the cubic torus). These groups are listed in Table 1 following the International Tables for Crystallography nomenclature; see [11] and the website [18. Primitive groups, named $P$..., do not have nonzero translations. Body-centered groups $I \ldots$ contain the body-centering translation $(x+1 / 2, y+1 / 2, z+1 / 2)$ and face-centered groups $F \ldots$ contain the face-centering translations $(x, y+1 / 2, z+1 / 2)$, $(x+1 / 2, y, z+1 / 2)$ and $(x+1 / 2, y+1 / 2, z)$.

Let $\Sigma \subset T$ be a surface enclosing a $\mathcal{G}$-invariant region $\Omega$. Our convention about the sign of the mean curvature and the principal curvatures of $\Sigma$ is the one which gives $H=1$ when $\Sigma$ is an unit sphere and $\Omega$ is the ball inside. We can consider the (single) equidistant surface $\Sigma_{t}=\{$ points in $T$ whose oriented distance to $\Sigma$ is $t\}$. The oriented distance to $\Sigma$ is measured with respect to the unit normal pointing towards $T-\Omega$ and $t$ is taken in the connected range around $t=0$ where $\Sigma_{t}$ 
TABLE 3 .

\begin{tabular}{cccc}
\hline$\Sigma$ & $\chi(\Sigma)$ & Area & First focal value \\
\hline Schwarz $P$ & -4 & $3 \mathrm{~K} / \mathrm{K}^{\prime}=2.3451$ & $1 /\left(2 \mathrm{~K}^{\prime}\right)=0.2318$ \\
Schwarz $D$ & -16 & $3 \mathrm{~K}^{\prime} / \mathrm{K}=3.8378$ & $1 /(4 \mathrm{~K})=0.1483$ \\
Schoen $G$ & -8 & $3 \mathrm{~K}^{\prime \prime} /\left(2 \mathrm{KK}^{\prime}\right)=3.0914$ & $\sqrt{\mathrm{K}^{\prime \prime}} /\left(4 \mathrm{KK}^{\prime}\right)=0.1882$ \\
\hline
\end{tabular}

is free of singularities. Any equidistant surface with $t \neq 0$ lies either in $\Omega$ or in $T-\Omega$ (corresponding to the cases $t<0$ and $t>0$, respectively). The double equidistant surface $\Sigma_{t} \cup \Sigma_{-t}$ consists of points in $T$ whose (unoriented) distance to $\Sigma$ is equal to $t$. Note that the region enclosed by $\Sigma_{t}$ has the same symmetries as $\Omega$. In general, the control of the range where the equidistant surface is nonsingular depends of global information about $\Sigma$. However in the case where $\Sigma$ has nonnegative mean curvature, as a consequence of the maximum principle, it depends only on the curvature of the surface.

Lemma 3 ([26]). If $\Sigma \subset T$ is nonplanar, bounds a region $\Omega$ and has nonnegative mean curvature $H \geq 0$ (towards the $\Omega$ side), then the equidistant surface $\Sigma_{t} \subset \Omega$ is nonsingular in the range $-t_{0}<t \leq 0$, where $t_{0}=1 /$ the largest positive principal curvature of $\Sigma$ \} is the first focal value of $\Sigma$ in $\Omega$. If $\Sigma$ is minimal and nonplanar, then the same holds at both sides of $\Sigma$.

If $\Sigma$ has constant mean curvature $H$, then the volume enclosed by the equidistant surface $\Sigma_{t}$ and its area can be expressed in term of the volume of $\Omega, V$, the area of $\Sigma, A$, its Euler characteristic $\chi$, and $H$. They are given by

$$
V(t)=V+A t+H A t^{2}+\frac{2 \pi}{3} \chi t^{3}, \quad A(t)=A+2 H A t+2 \pi \chi t^{2} .
$$

The area and the first focal value of the minimal $P$ and $D$ Schwarz surfaces and the Schoen Gyroid $G$ (in the unit cubic torus) can be computed after their Weierstrass data; see for instance [7. They are given in Table 3 in term of the elliptic integrals $\mathrm{K}=$ EllipticK $[1 / 4]$ and $\mathrm{K}^{\prime}=$ EllipticK $[3 / 4]$ and $\mathrm{K}^{\prime \prime}=\mathrm{K}^{2}+\left(\mathrm{K}^{\prime}\right)^{2}$. To compute the first focal value of the $P$ surface, use that the maximum of the absolute Gauss curvature is attained at the intersection with the 2 -fold axes (along the $\langle 100\rangle$ directions). For the other surfaces use that the three minimal surfaces $P$, $D$ and $G$ lie in the same Bonnet family (up to a positive factor) ([15).

Consider the following family of ordinary differential equations, parameterized by an even integer number $\chi$ : $\star \chi$

$$
J^{2} J^{\prime \prime}+J\left(J^{\prime}\right)^{2}-4 \pi \chi=0
$$

Lemma 4 ([12]). Let $J$ be a (maximal) nonnegative solution of $\star \chi$, with $J>0$ at some point. Assume $\chi<0$.

(i) If $J$ verifies the initial conditions $J\left(V_{0}\right)=A_{0}$ and $J^{\prime}\left(V_{0}\right)=2 H_{0}$, then the graph of $J$ is parameterized by the curve

$$
\gamma(t)=\left(V_{0}+A_{0} t+H_{0} A_{0} t^{2}+\frac{2 \pi}{3} \chi t^{3}, A_{0}+2 H_{0} A_{0} t+2 \pi \chi t^{2}\right),
$$

where $t_{1} \leq t \leq t_{2}$, for certain $t_{1}, t_{2}$. 
(ii) $J$ is concave, symmetric with respect to a vertical line and meets the horizontal axis orthogonally.

Note that if $\Sigma \subset T$ is a closed surface with $\chi(\Sigma)=\chi$, area $A_{0}$, enclosing a region of volume $V_{0}$ and with mean curvature $H_{0}$, then it follows from (4) that the curve $\gamma(t)$ describes the volume-area of the equidistant surface $\Sigma_{t}$, if we restrict $t$ to the connected range around $t=0$ where this equidistant surface is nonsingular.

The only solutions of $\star \chi$ we will use in the case $\chi \geq 0$ will be the volume-area profile of $k$ round balls (of the same radius) $J_{k}(v)=\left(36 k \pi v^{2}\right)^{1 / 3}$ (with $\chi=2 k$ ) or the complementary regions in $T, J_{k}(1-v)$, or the volume-area profile of $n$ closed cylinders in $T$ all related by symmetries (with $\chi=0$ ).

The $\mathcal{G}$-isoperimetric problem consists of minimizing the area among closed surfaces $\Sigma \subset T$ enclosing a $\mathcal{G}$-invariant region of prescribed volume. These least area surfaces (resp. the regions they enclose) are called $\mathcal{G}$-isoperimetric surfaces (resp. $\mathcal{G}$ isoperimetric regions). Isoperimetric surfaces $\Sigma$ always exist and they are smooth surfaces (without singularities) with constant mean curvature; see [3, 8, 20] and 12, 21] for the necessary modifications for adapting the general result to the symmetric case. Although $G$-isoperimetric surfaces do not need to be connected, we have that either $\Sigma$ is planar or the connected components of $\Sigma$ are all related by the symmetries of $\mathcal{G}$.

Isoperimetric surfaces with nonnegative Euler characteristic can be described completely. The following facts were proved in [12] by using results from [22, 25, 27. (the planar case appears as another possibility in Theorem 8 of [12] but not here since it is clearly incompatible with the cubic symmetry).

Proposition 5. Let $\Sigma \subset T$ be a $\mathcal{G}$-isoperimetric surface enclosing a volume $v$.

(i) If $\chi(\Sigma)>0$, then $\Sigma$ is a $\mathcal{G}$-invariant family of round spheres. This holds in particular if $v$ is small enough.

(ii) If $\chi(\Sigma)=0$, then $\Sigma$ is a $\mathcal{G}$-invariant family of right cylinders.

The $\mathcal{G}$-isoperimetric profile is the function $I_{\mathcal{G}}$ which assigns to each volume $v$, $0<v<1$, the area of $\mathcal{G}$-isoperimetric surfaces $\Sigma$ enclosing a volume $v$. The profile $I_{\mathcal{G}}$ is symmetric , $I_{\mathcal{G}}(v)=I_{\mathcal{G}}(1-v)$, concave (in particular $I_{\mathcal{G}}$ has lateral derivatives everywhere) and it attains its maximum at $v=1 / 2$.

Moreover the profile $I_{\mathcal{G}}$ satisfies (in the weak sense) the differential inequality

$$
I_{\mathcal{G}}^{2} I_{\mathcal{G}}^{\prime \prime}+I_{\mathcal{G}}\left(I_{\mathcal{G}}^{\prime}\right)^{2}-4 \pi \chi(\Sigma) \leq 0 \quad \text { at } \quad v,
$$

where $\chi(\Sigma)$ is the Euler characteristic of $\Sigma$; see 4]. This means that if we consider the solution $J$ of $\star \chi$ with $\chi=\chi(\Sigma), J(v)=A(\Sigma)$ and $J^{\prime}(v)=2 H$ in a small interval around $v$, then we have $J \geq I_{\mathcal{G}}$ in that interval. The inequality follows because $J$ is the volume-area profile of the equidistant surfaces to $\Sigma$ and $I_{\mathcal{G}}$ is the minimizing volume-area profile among $\mathcal{G}$-invariant surfaces in $T$. Thus $I_{\mathcal{G}}$ is a supersolution of $\star \chi$.

The mean curvature $H$ of $\Sigma$ is controlled by the slope (i.e., by the lateral derivatives) of $I_{\mathcal{G}}$ at $v$ : $\left(I_{\mathcal{G}}\right)_{-}^{\prime}(v) \leq 2 H \leq\left(I_{\mathcal{G}}\right)_{+}^{\prime}(v)$; see [4] 12, 27]. As a consequence, if a minimal surface $\Sigma$ is isoperimetric, then $\Sigma$ divides the torus $T$ into two equal volumes. This can be seen as follows: otherwise the profile will be constant along an open interval. From (6), that would be possible only if any isoperimetric surface in that range has nonnegative Euler characteristic, but this contradicts Proposition 5 which says that there are no minimal surfaces satisfying this condition. We also 
have that

$$
H>0 \quad(\text { resp. } H<0) \quad \text { implies } \quad v \leq \frac{1}{2} \quad\left(\text { resp. } v \geq \frac{1}{2}\right) .
$$

Proposition 6 (The maximum principle, 12]). Let $I$ and $J$ be a nonnegative solution of the equation $\star \chi$ and a nonnegative supersolution of $\star \chi^{\prime}$, respectively, both defined in the interval $\left[v_{1}, v_{2}\right]$. Assume that $\chi^{\prime} \leq \min \{0, \chi\}$.

(i) If $I\left(v_{1}\right) \leq J\left(v_{1}\right)$ and $I\left(v_{2}\right) \leq J\left(v_{2}\right)$, then $I \leq J$ in $\left[v_{1}, v_{2}\right]$. If the equality holds at some interior value, then $I=J$ in the whole interval.

(ii) If $I\left(v_{1}\right) \geq J\left(v_{1}\right)$ and $I^{\prime}\left(v_{1}\right) \geq J_{+}^{\prime}\left(v_{1}\right)$, then $I-J$ is nondecreasing in $\left[v_{1}, v_{2}\right]$.

The following result (see Theorem 8 in [12]) shows that only for spheres and cylinders can equality hold in (6)).

Lemma 7. If $I_{\mathcal{G}}$ satisfies the equation $\star \chi$ in an open interval, $v_{1}<v<v_{2}$, $\chi$ being an upper bound of the Euler characteristic of $\mathcal{G}$-isoperimetric surfaces in that range, then any isoperimetric surface enclosing a volume in the interval $\left(v_{1}, v_{2}\right)$ must be either spherical or cylindrical.

For properties of triply periodic minimal surfaces see [15] and for general facts about the isoperimetric problem see [27].

\section{ISOPERIMETRY AND SYMMETRIES}

Theorem 8. Given a cubic space group $\mathcal{G}$, the $\mathcal{G}$-isoperimetric problem in the cubic torus $T=\mathbb{R}^{3} / \mathbb{Z}^{3}$ satisfies the following properties:

(i) Isoperimetric surfaces with positive Euler characteristic consist of $k=k(\mathcal{G})$ spheres centered at one of the smallest orbits of $\mathcal{G}$. Moreover there is a positive constant $v_{\text {shp }}=v_{\text {shp }}(\mathcal{G})$ such that the above spheres are isoperimetric if and only if they enclose a volume $v \leq v_{\text {shp }}$ (or $1-v_{\text {sph }} \leq v<1$ ).

(ii) There are no isoperimetric surfaces $\Sigma \subset T$ with $\chi(\Sigma)=0$.

(iii) If $\mathcal{T}$ is the translations subgroup of $\mathcal{G}$ and $\Sigma \subset T$ is a $\mathcal{G}$-isoperimetric surface with $\chi(\Sigma)<0$, then $\chi(\Sigma) \leq-4|\mathcal{T}|$. In particular $\chi(\mathcal{G}) \leq-4$.

Proof. Recall that $J_{k}(v)=\left(36 k \pi v^{2}\right)^{1 / 3}$ is the volume-area profile of $k$ equal spheres. We know that any $\mathcal{G}$-isoperimetric surface enclosing a small enough volume consists of $k$ spheres (centered at one of the smallest orbits of $\mathcal{G}$ ) by Proposition 5 ; for details see [22, 12]. Let $v_{s p h}, 0<v_{s p h} \leq 1 / 2$, be the largest volume such that $I_{\mathcal{G}}(v)=J_{k}(v)$, for $v \leq v_{s p h}$. If spheres where isoperimetric for some volume between $v_{s p h}$ and $1 / 2$, then we would have that $J_{k}$ and $I_{\mathcal{G}}$ coincide at $v_{s p h}$ and $v_{1}$, with $v_{s p h}<v_{1} \leq 1 / 2$. Between $v_{s p h}$ and $v_{1}$ the profile $I_{\mathcal{G}}$ is a supersolution of $I^{2} I^{\prime \prime}+I\left(I^{\prime}\right)^{2} \leq 0$ and $J_{k}$ is a solution of $I^{2} I^{\prime \prime}+I\left(I^{\prime}\right)^{2}-8 k \pi=0$. Moreover $I_{\mathcal{G}}<J_{k}$ at the interior of that interval. From the maximum principle in Proposition [6 we conclude that $I_{\mathcal{G}} \geq J_{k}$ for $v_{s p h} \leq v \leq v_{1}$ and this contradiction proves (i).

Now we prove (ii). Let $\Sigma \subset T$ be a $\mathcal{G}$-isoperimetric surface whose connected components are 2 -tori. These tori must be nonintersecting right cylinders all related by symmetry by Proposition [5. These figures have been studied by O'Keeffe et al. [23, 24]. If the axes of these cylinders are neither rotation axes nor screw axes, then each cylinder can be translated suitably normal to its axis in such a way that the new surface $\Sigma^{\prime}$ is still $\mathcal{G}$-invariant and, thus, $\Sigma^{\prime}$ is $\mathcal{G}$-isoperimetric, too. We keep on translating until two components of $\Sigma^{\prime}$ touch each other for the first time (that will certainly hold before the cylinders meet some of the 3 -fold axes) which 
contradicts the regularity properties of isoperimetric surfaces. Therefore, the axes of the cylinders of $\Sigma$ are parallel either to $\langle 100\rangle$ or $\langle 110\rangle$ or $\langle 111\rangle$ directions (these directions correspond to the edges of the cube, the diagonal of its faces and the body diagonals, respectively).

In the case $\langle 110\rangle$ the axes of each cylinder will be perpendicular to one of the 3 -fold axes and so the cylinder will intersect its rotated image. Therefore there are no nonintersecting $\mathcal{G}$-invariant $\langle 110\rangle$-cylinders $(23])$.

In the remaining two cases, we will exclude most (but not all) of the cubic groups by direct comparison of cylindrical and spherical $\mathcal{G}$-invariant surfaces. The surface $\Sigma$ consists of $n$ cylinders of radius $r$ and length of its axis $l$. Hence the area of $\Sigma$ is $A=$ $2 \pi n l r$ and the enclosed volume $V=\pi n l r^{2}$. Since $\Sigma$ does it better than $k$ spheres enclosing the same volume, we have $36 k \pi \geq A^{3} / V^{2}=8 \pi^{3} n^{3} l^{3} r^{3} /\left(\pi^{2} n^{2} l^{2} r^{4}\right)=$ $8 \pi n l / r$ or, equivalently,

$$
9 k r \geq 2 n l .
$$

To obtain an upper bound for $r$, we use that, because of (7), we have $1 / 2 \geq V=$ $n l \pi r^{2}$, which gives $r^{2} \leq 1 /(2 n l \pi)$. From (88) we obtain

$$
\frac{81 k^{2}}{2 n l \pi} \geq(9 k r)^{2} \geq(2 n l)^{2},
$$

and we conclude

$$
k^{2} \geq \frac{8 \pi l^{3} n^{3}}{81} .
$$

Now we discuss the two cases separately.

Case $\langle 100\rangle$. We have $l=1$. Symmetry and embeddedness imply that $n=$ $3,6, \ldots$ If $n=3$, then there is only a cubic family of $\langle 100\rangle$-cylinders; see 23.24 . The axes of these cylinders are the lines $(1 / 2,0, t),(t, 1 / 2,0)$ and $(0, t, 1 / 2)$ and the family has symmetry $P m \overline{3} n$. Therefore $\mathcal{G}$ must be a primitive subgroup of this group (i.e., $\mathcal{G}=P m \overline{3} n, P \overline{4} 3 n, P m \overline{3}, P 4_{2} 32$ or $P 23$ ) and, in particular, $k \leq 2$ (see Table (1) which contradicts the inequality (9). If $n \geq 6$, then (9) give $k>8$; that is, if the smallest orbit of $\mathcal{G}$ has $k \leq 8$ points, then $\langle 100\rangle$-cylinders with $n \geq 6$ are not isoperimetric. This exclude all the primitive cubic groups; see Table1.

If $\mathcal{G}$ is a body-centered group, then using the centering translation $(1 / 2,1 / 2,1 / 2)$, we get that $n=6,12, \ldots$, which together with (9) implies $n=6$ and $k>8$. Using Table 1] we conclude that $\mathcal{G}$ must be either $I \overline{4} 3 d$ or $I a \overline{3} d$. According to [23, 24] there is a unique (up to enantiomorphisms) invariant cubic family of $\langle 100\rangle$-cylinders with $n=6$. Since this family has symmetry $I 4_{1} 32$, we exclude these groups too.

In the same way, if $\mathcal{G}$ is face-centered, then the invariance under the translations $(1 / 2,1 / 2,0),(1 / 2,0,1 / 2),(0,1 / 2,1 / 2)$ imply $n \geq 12$ and, using (9)), we contradict $k \leq 16$ in Table 1

Case $\langle 111\rangle$. We have $l=\sqrt{3}$ and $n=4,8, \ldots$ From (9) we obtain $k^{2} \geq$ $2^{3} 3^{-5 / 2} \pi n^{3}>100$. Therefore, if $k \leq 10$, there are not $\mathcal{G}$-isoperimetric cylinders of type $\langle 111\rangle$. This argument excludes $\langle 111\rangle$ isoperimetric cylinders in all the cases except for three groups: $I \overline{4} 3 d, I a \overline{3} d$ and $F d \overline{3} c$ which have $k=12$ or 16 . Moreover, for these groups we necessarily have $n=4$.

Since the group $F d \overline{3} c$ contains the glide symmetry $\sigma:(x, y, z) \mapsto(y, x, z+1 / 2)$, there are not embedded $\langle 111\rangle$-cylinders with $F d \overline{3} c$ symmetry (the one whose axis has the direction $(1,-1,1)$ will intersects its image under $\sigma)$. 
Finally there is a (unique) figure $\Gamma$ of $\langle 111\rangle$-cylinders with $\operatorname{Ia} \overline{3} d$ (or $I \overline{4} 3 d$ ) symmetry and $n=4$ (see $[23,24]$ ), the axes of the cylinders being just the 3 -fold axes of the group $(t, t, t),(-t, 1 / 2-t, t),(1 / 2+t,-t, t)$ and $(1 / 2-t, 1 / 2+t, t)$. It encloses volumes between 0 and $3^{1 / 2} \pi / 8=0.680$. Since $I \overline{4} 3 d$ is an index 2 subgroup of $I a \overline{3} d$, to finish the proof of item (ii), it is enough to reason that $\Gamma$ is not $I a \overline{3} d-$ isoperimetric. We will do that by comparison with (equidistant) double Gyroids $G_{r}$ which consist of points whose distance to the minimal Gyroid $G$ is equal to $r$. From Lemma 3 and Table 3 we have that the double Gyroid $G_{r}$ exists for $0<r<0.188$. It has two congruent components and $\chi\left(G_{r}\right)=2 \chi(G)=-16$. The area of $G_{r}$ and the volume of the (mean convex) enclosed region $\{x \in T \mid \operatorname{dist}(x, G) \geq r\}$ are given (see (4)) by

$$
A(r)=2\left(A_{1}-16 \pi r^{2}\right), \quad V(r)=2\left(\frac{1}{2}-A_{1} r+\frac{16 \pi}{3} r^{3}\right),
$$

where $A_{1}=3.0914 \ldots$ is the area of the minimal $G$ surface. The graph $A=J(V)$ of the curve $(V(r), A(r))$ verifies the differential equation $J^{2} J^{\prime \prime}+J\left(J^{\prime}\right)^{2}-64 \pi=0$.

For the four cylinders in $\Gamma$ (resp. for 16 spheres) the volume-area profile is $A=$ $4\left(3^{1 / 2} \pi V\right)^{1 / 2}\left(\right.$ resp. $\left.A=\left(16 \times 36 \pi V^{2}\right)^{1 / 3}\right)$. These profiles meet at $V=0.201 \ldots$ and the first one is smaller than the second one if and only if $V$ is greater than this value.

For $r_{1}=0.154$ the point $(V(r), A(r))$ is $(0.170 \ldots, 3.798 \ldots)$ which lies below the $\Gamma$ volume-area profile and the slope of $A=J(V)$ at that point is 8.15129, which is smaller than the slope of the $\Gamma$-profile at the same $V$. Thus the maximum principle ((ii) of Proposition 6) implies that $J(V)$ is smaller than the $\Gamma$-profile for $0.171<V \leq 0.5$. That proves (ii).

To prove (iii), we use particular properties of genus two surfaces. Let $\Sigma^{\prime}=\Sigma / \mathcal{T}$ be the quotient surface in the 3 -torus $T^{\prime}=T / \mathcal{T}$ and suppose that $\chi\left(\Sigma^{\prime}\right)=-2$. We know from Meeks [19] that the mean convex region enclosed by $\Sigma^{\prime}$ is a (genus two) handlebody. Therefore the connected components of the full lift $\widetilde{\Sigma}$ of $\Sigma^{\prime}$ to $\mathbb{R}^{3}$ are at most doubly periodic. If these components where either nonperiodic or singly periodic, then, because of special properties of embedded constant mean curvature surfaces [1, 16], they will be round spheres or Delaunay cylinders, which is impossible. Thus the components of $\widetilde{\Sigma}$ are doubly periodic constant mean curvature surfaces and so they are contained in a (unique) slab of minimal width; see for instance 12. Since these components differ from each other by a translation, these slabs are all parallel, which contradicts the cubic symmetry of the surface. Therefore $\chi\left(\Sigma^{\prime}\right) \leq-4$ and we have proved the theorem.

Now we prove that the isoperimetric profile $I_{\mathcal{G}}$ of a cubic space group $\mathcal{G}$ is bounded below by the function $J_{k, \chi}, I_{\mathcal{G}} \geq J_{k, \chi}$, where $k=k(\mathcal{G})$ and $0>\chi \geq \chi(\mathcal{G})$.

Proof of Theorem Q Denote by $\Gamma_{A}, A>0$, the trace of the curve $\gamma_{A}(t)=(v(t), a(t))$, with $v(t)=1 / 2+A t+2 \pi \chi t^{3} / 3, a(t)=A+2 \pi \chi t^{2}$ and $t \in \mathbb{R}$, intersected with the upper half plane $a \geq 0$. From (5) we have that $\Gamma_{A}$ is the graph of the solution of the equation $J^{2} J^{\prime \prime}+J\left(J^{\prime}\right)^{2}-4 \pi \chi=0$ satisfying the initial conditions $J(1 / 2)=A$ and $J^{\prime}(1 / 2)=0$. Moreover $\Gamma_{A}$ is concave, has a mirror symmetry at $v=1 / 2$ and meets the $v$-axis orthogonally at two points which converge to $(1 / 2,0)$ (resp. $( \pm \infty, 0))$ when $A$ goes to zero (resp. infinity). 
Thus for small values of $A$ the curve $\Gamma_{A}$ lies below the isoperimetric profile $I_{\mathcal{G}}$. If we denote by $A_{0}$ the largest value of $A$ satisfying this property, then we must have a first contact between $\Gamma_{A_{0}}$ and the graph of $I_{\mathcal{G}}$. This contact cannot hold at $v=0$, since the curves $\Gamma_{A}$ meet the $v$-axis orthogonally and $I_{\mathcal{G}}(v)=J_{k}(v)=\left(36 k \pi v^{2}\right)^{1 / 3}$ for $v$ small enough, nor at the (open) nonspherical range $v_{s p h}(\mathcal{G})<v<1-v_{s p h}(\mathcal{G})$, by the maximum principle in Proposition 6 and Lemma 7 If the first contact holds at $v=v_{s p h}$, then $\Gamma_{A_{0}}$ and $J_{k}$ meet tangentially at $v_{s p h}(\mathcal{G})$ and $I_{\mathcal{G}}$ is trapped between them, $\Gamma_{A_{0}} \leq I_{\mathcal{G}} \leq J_{k}$, for $v_{s p h} \leq v \leq 1 / 2$, which is impossible too, by the boundary version of the maximum principle in Proposition 6

Therefore the first contact between the curves $\Gamma_{A}, A>0$, and $I_{\mathcal{G}}$ holds necessarily at some value $v_{0}$ in the (open) spherical range $0<v_{0}<v_{s p h}(\mathcal{G})$ (and its symmetric value with respect to $v=1 / 2$ ). This value is characterized by the fact that there is a solution of $\star \chi$ which meets the $k$ spheres profile $J_{k}$ tangentially at $v_{0}$ and attains its maximum at $v=1 / 2$. Moreover the $C^{1}((0,1))$ graph given by $\Gamma_{A_{0}}\left(\right.$ for $\left.v_{0} \leq v \leq 1-v_{0}\right), J_{k}(v)\left(\right.$ for $\left.0 \leq v \leq v_{0}\right)$ and $J_{k}(1-v)\left(\right.$ for $\left.1-v_{0} \leq v \leq 1\right)$ is a lower bound of the isoperimetric profile $I_{\mathcal{G}}$. To prove that this curve is just the graph of the function $J_{k, \chi}$, we only need to compute $v_{0}$ and $A_{0}$.

To do that, we take a generic point $\left(4 k \pi r^{3} / 3,4 k \pi r^{2}\right), r>0$, in the graph of $J_{k}$ and we consider the solution $J$ of $\star \chi$ starting at that point with the same slope as $J_{k}$. Since this slope is $2 / r$, by using (5), we obtain that $J$ is given by the graph of the curve

$$
\gamma(s)=\left(\frac{4}{3} k \pi r^{3}+4 k \pi r^{2} s+4 k \pi r s^{2}+\frac{2}{3} \pi \chi s^{3}, 4 k \pi r^{2}+8 k \pi r s+2 \pi \chi s^{2}\right), s \geq 0 .
$$

The highest point of this curve is attained for $s_{0}=-2 k r / \chi$ and its value is

$$
\gamma\left(s_{0}\right)=\left(\frac{4 k \pi}{3 \chi^{2}}(4 k-\chi)(2 k-\chi) r^{3}, \frac{4 k \pi}{\chi}(\chi-2 k) r^{2}\right) .
$$

If we impose the condition that this point lies on the line $v=1 / 2$, we obtain that $r$ must be equal to

$$
r_{0}=\left(\frac{3 \chi^{2}}{8 k \pi(4 k-\chi)(2 k-\chi)}\right)^{1 / 3} .
$$

For $r=r_{0}$ the curve $\gamma(s)$ is just a reparametrization of $\gamma_{A_{0}}(t)$ with $t=s-s_{0}$. As a consequence we have that

$$
\begin{gathered}
s_{0}=-2 k r_{0} / \chi=\left(\frac{3 k^{2}}{\pi(-\chi)(4 k-\chi)(2 k-\chi)}\right)^{1 / 3}, \\
v_{0}=\text { the first component of } \gamma(0)=\frac{\chi^{2}}{2(4 k-\chi)(2 k-\chi)},
\end{gathered}
$$

and

$$
A_{0}=\text { the second component of } \gamma\left(s_{0}\right)=\left(\frac{9 k \pi(-\chi)(2 k-\chi)}{(4 k-\chi)^{2}}\right)^{1 / 3} .
$$

That conclude the proof of the theorem.

Theorem 1 follows as a direct consequence of Theorem 2 and (12). It follows also from the proof of Theorem 2 that the nonspherical part of $J_{k, \chi}$ can be parameterized by $\gamma_{A_{0}}(t)$, as in (3). In particular, the range of variation of the parameter $t$ is given by $|t| \leq s_{0}$. 


\section{Cubic space groups}

In this section we explain the properties we need for the different kind of cubic groups acting on the cubic torus $T=\mathbb{R}^{3} / \mathbb{Z}^{3}$. Given a cubic group $\mathcal{G}$, we will denote by $k=k(\mathcal{G})$ (resp. $\chi=\chi(\mathcal{G})$ ) the smallest number of points in a $\mathcal{G}$-orbit (resp. the largest Euler characteristic among nonspherical $\mathcal{G}$-isoperimetric surfaces), and $\Sigma \subset T$ will be a nonspherical $\mathcal{G}$-isoperimetric surface. The value of $k$ can be obtained by looking at the Wyckoff positions (in crystallography, Wyckoff positions describe the different types of orbits of the group) in the Crystallographic Tables; see 11 18.

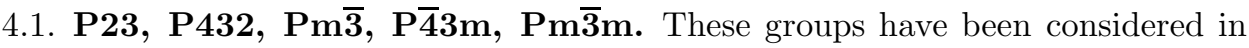
12. They have $k=1$ (the fixed point being either $(0,0,0)$ or $(1 / 2,1 / 2,1 / 2)$ ) and $\chi \leq-4$. The Primitive $P$ Schwarz surface is a natural candidate for solving the isoperimetric problem which has Euler characteristic equal to -4 . We have conjectured that all the five groups have the same isoperimetric surfaces which should be either spherical or $P$ Schwarz surfaces.

4.2. $\mathbf{P} 4_{2}$ 32, $\mathbf{P} \overline{\mathbf{4}} \mathbf{3}, \mathbf{P n} \overline{\mathbf{3}}, \mathbf{P n} \overline{\mathbf{3}} \mathbf{m}, \mathbf{P m} \overline{\mathbf{3}} \mathbf{n}, \mathbf{P n} \overline{\mathbf{3}} \mathbf{n}$. The smallest orbit is given by $(0,0,0),(1 / 2,1 / 2,1 / 2)$. As a consequence of Theorem 8 , isoperimetric surfaces for these groups are either a couple of spheres, for small volumes, or surfaces with $\chi \leq-4$, for volumes close than $1 / 2$.

In the case $\operatorname{Pn} \overline{3} m$ we have a natural candidate for solving the isoperimetric problem, the double $D$, whose Euler characteristic is -4 .

4.3. $\mathbf{P} 4_{1} 32\left(\mathbf{P} 4_{3} 32\right), \mathbf{P a} \overline{3}, \mathbf{P 2}_{1}$ 3. Each of these groups have the subgroup $\mathcal{G}^{\prime}=$ $P 2_{1} 2_{1} 2_{1}$ given by the screw involutions $(x, y, z),(-x+1 / 2,-y, z+1 / 2),(-x, y+$ $1 / 2,-z+1 / 2)$ and $(x+1 / 2,-y+1 / 2,-z)$. The action of $\mathcal{G}^{\prime}$ is free and, so, the quotient $T / \mathcal{G}^{\prime}$ is a flat 3 -manifold and $\Sigma / \mathcal{G}^{\prime}$ is an orientable compact surface with negative Euler characteristic. Hence, $\chi(\Sigma)=4 \chi\left(\Sigma / \mathcal{G}^{\prime}\right) \leq-8$, which implies $\chi(\mathcal{G}) \leq-8$.

4.4. I23, I432, Im $\overline{3}, \mathbf{I} \overline{\mathbf{4}} \mathbf{3} \mathbf{m}, \mathbf{I m} \overline{\mathbf{3}} \mathbf{m}$. A body-centered group $\mathcal{G}$ contains the translation $(1 / 2,1 / 2,1 / 2)$ and thus, from Theorem 8 the Euler characteristic of a nonspherical isoperimetric surface in the torus $T$ is at most -8 . So $\chi(\mathcal{G}) \leq-8$.

For the groups in this paragraph, we have $k(\mathcal{G})=2$ and the smallest orbit is given by the points $(0,0,0)$ and $(1 / 2,1 / 2,1 / 2)$. Natural candidates for solving the isoperimetric problem for the group $\operatorname{Im} \overline{3} m$ are the double constant mean curvature Schwarz $P$ surfaces $(\chi=-8)$ and the (constant mean curvature) $I-W P$ surfaces of Schoen $(\chi=-12)$. All the five groups are conjectured to have the same isoperimetric surfaces.

4.5. I $\mathbf{2}_{\mathbf{1}} \mathbf{3}, \mathbf{I} \mathbf{I}_{\mathbf{1}} \mathbf{3 2}$. As in $\S 4.3$, these groups have the order four subgroup $P 2_{1} 2_{1} 2_{1}$ and therefore $\chi(\Sigma) \leq-2 \times 4=-8$. The smallest orbit has 8 points (the union of two $P 2_{1} 2_{1} 2_{1}$-orbits). The minimal $G$ surface of Schoen and its constant mean curvature deformations (see for instance [9]) are natural candidates for the isoperimetric problem. They have Euler characteristic equal to -8 . 
4.6. Ia $\overline{3}, \mathbf{I a} \overline{3} \mathbf{d}, \mathbf{I} \overline{\mathbf{4}} \mathbf{3} \mathrm{d}$. To find a fine estimate of $\chi$ in this case, we will consider the group $\mathcal{G}=I a \overline{3}$ in details. This group has 2 -fold and 3 -fold axes and it has no mirror symmetries. If we take the quotient by the body-centering translation $\tau=(1 / 2,1 / 2,1 / 2)$, we get a group $\mathcal{G}^{\prime}=\mathcal{G} / \tau$ of order 24 acting on the torus $T^{\prime}=T / \tau$ and an orientable surface $\Sigma^{\prime}=\Sigma / \tau \subset T^{\prime}$ enclosing a $\mathcal{G}^{\prime}$-invariant region. The 2 -fold axes of $\mathcal{G}^{\prime}$ are given by the Wyckoff position $d$ :

$$
\begin{array}{llll}
\left(x, 0, \frac{1}{4}\right) & \left(x+\frac{1}{2}, 0, \frac{1}{4}\right) ; & \left(-x, 0, \frac{3}{4}\right) & \left(-x+\frac{1}{2}, 0, \frac{3}{4}\right) ; \\
\left(\frac{1}{4}, x, 0\right) & \left(\frac{1}{4}, x+\frac{1}{2}, 0\right) ; & \left(\frac{3}{4},-x, 0\right) & \left(\frac{3}{4},-x+\frac{1}{2}, 0\right) ; \\
\left(0, \frac{1}{4}, x\right) & \left(0, \frac{1}{4}, x+\frac{1}{2}\right) ; & \left(0, \frac{3}{4},-x\right) & \left(0, \frac{3}{4},-x+\frac{1}{2}\right) .
\end{array}
$$

Hence, it has 6 nonintersecting 2-fold axes, all related by symmetry. Moreover the group induces on each of these axes an involution without fixed points (like a central symmetry on a circle). Therefore the number of 2 -fold centers on the surface $\Sigma^{\prime}$, under the action of $\mathcal{G}^{\prime}$, is given by $6 \times 4 \times n_{2}$, for some nonnegative integer $n_{2}$.

Wyckoff site $c$ corresponds to the 3 -fold axes:

$$
\begin{array}{ccc}
(x, x, x) & (-x,-x,-x) ; \\
\left(x+\frac{1}{2}, x,-x+\frac{1}{2}\right) & \left(-x+\frac{1}{2},-x, x+\frac{1}{2}\right) ; \\
\left(-x, x+\frac{1}{2},-x+\frac{1}{2}\right) & \left(x,-x+\frac{1}{2}, x+\frac{1}{2}\right) ; \\
\left(x+\frac{1}{2},-x+\frac{1}{2},-x\right) & \left(-x+\frac{1}{2}, x+\frac{1}{2}, x\right) .
\end{array}
$$

So, the group $\mathcal{G}^{\prime}$ has 4 nonintersecting 3 -fold axes, all related by symmetry, and each of these axes has a symmetry involution with two fixed points (like a mirror symmetry on a circle). Moreover 2 -fold axes and 3-fold axes do not meet. Thus the number of 3 -fold centers on the surface $\Sigma^{\prime}$ above are $4 \times 2 \times n_{3}$, for some $n_{3}$. When we pass to the double covering, we obtain that the group $\mathcal{G}$ acting on the surface $\Sigma$ has $2 \times 24 n_{2} 2$-fold centers and $2 \times 8 n_{3} 3$-fold centers. The Riemann-Hurwitz formula, relating the Euler characteristic of $\Sigma$ and the quotient Riemann surface $\Sigma / \mathcal{G}$, gives

$$
\chi(\Sigma)=2\left(24 \chi(\Sigma / \mathcal{G})-24 n_{2}-16 n_{3}\right) .
$$

Hence, the largest negative value admissible for the Euler characteristic of $\Sigma$ is $\chi(\Sigma)=-16$ (corresponding to $\chi(\Sigma / \mathcal{G})=2, n_{2}=1$ and $\left.n_{3}=2\right)$. In conclusion $\chi(\operatorname{Ia} \overline{3}) \leq-16$ and the same holds for its supergroup $I a \overline{3} d$. With the same kind of arguments one can show that $\chi(I \overline{4} 3 d) \leq-16$. Note that both $I a \overline{3}$ and $I \overline{4} 3 d$ are index 2 subgroups of $I a \overline{3} d$.

The double (constant mean curvature) Gyroid is a natural candidate for solving the $I a \overline{3} d$-isoperimetric problem which has just this topology.

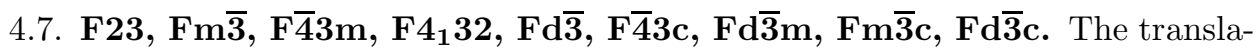
tions subgroup of a face-centered group $\mathcal{G}$ is given by the four involutions $(0,0,0)$, $(1 / 2,1 / 2,0),(1 / 2,0,1 / 2),(0,1 / 2,1 / 2)\left(\bmod \mathbb{Z}^{3}\right)$. From (iii) in Theorem 8 , we know that $\chi(\Sigma) \leq-4 \times 4$ and, thus, for any face-centered group we have $\chi(\mathcal{G}) \leq-16$. In 
the case $F d \overline{3} m$, we have a natural candidate for solving the isoperimetric problem with $\chi=-16$ : the Diamond $D$ Schwarz surface, [2, 15].

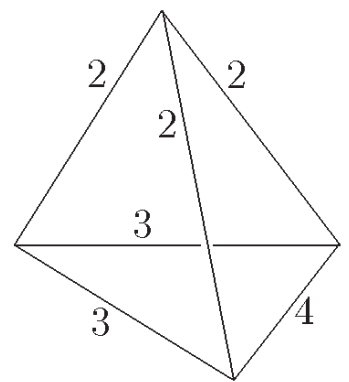

Figure 5 . The quotient orbifold $T / F 432$ of the cubic torus by the group $F 432$ is a 3 -sphere and its singular graph is given by the 6 edges of a tetrahedron. These edges correspond to the quotient of the 2-fold, 3-fold and 4-fold axes of the group.

4.8. $\mathbf{F} 432, \mathbf{F m} \overline{3} \mathbf{m}$. In this case we can improve the estimate given $\S 4.7$ by considering specific properties of these groups. Instead of reasoning as in $\S 4.6$, we use here the quotient orbifold. The order of $\mathcal{G}=F 432$ is 96 and the orbifold $T / \mathcal{G}$ is the 3 -sphere with a singular graph described in Figure 5 (the orbifolds of the cubic space groups are described in [14, 5]). Denote by $\chi^{\prime}=\chi(\Sigma / \mathcal{G})$ and $n_{i}, i=2,3,4$, the number of times the surface $\Sigma / \mathcal{G}$ intersects the $i$-fold axes (note that $\Sigma / \mathcal{G}$ does not meet the vertices of the tetrahedron). Each one of these intersection points produces a branch point of order $i-1$. Therefore, the Riemann-Hurwitz formula gives $\chi(\Sigma)=96 \chi^{\prime}-48 n_{2}-2 \times 32 n_{3}-3 \times 24 n_{4}$. If $\chi^{\prime} \leq 0$, we get $\chi(\Sigma) \leq-48$. If $\chi^{\prime}=2$, we have

$$
\chi(\Sigma)=192-48 n_{2}-64 n_{3}-72 n_{4}=8\left(24-6 n_{2}-8 n_{3}-9 n_{4}\right) .
$$

By checking the various possibilities, one can see that the largest value for $\chi(\Sigma)$ smaller than or equal to -16 is -32 , which corresponds to $\left(n_{2}, n_{3}, n_{4}\right)$ equal to $(2,2,0)$ (from Figure 5 use that we have (a) $n_{3}=0 \Rightarrow n_{4}$ is even, (b) $n_{3}=1 \Rightarrow$ $n_{4} \neq 0$ and (c) $n_{2}=0 \Rightarrow n_{3}$ and $n_{4}$ are even). Thus $\chi(F 432) \leq-32$. The argument applies to the supergroup $F m \overline{3} m$.

\section{REFERENCES}

[1] A. D. Alexandrov, Uniqueness theorems for surfaces in the large I, Vestnik Leningrad Univ. Math. 11 (1956), 5-17. MR 19:167c

[2] D. M. Anderson, H. T. Davis, J. C. C. Nitsche, and L. E. Scriven, Periodic Surfaces of Prescribed Mean Curvature, Advances in Chemical Physics 77 (1990), 337-396.

[3] F. J. Almgren, Existence and regularity almost everywhere of solutions to elliptic variational problems with constraints, Mem. AMS 165 (1976). MR 54:8420

[4] C. Bavard and P. Pansu, Sur le volume minimal de $\mathbf{R}^{2}$, Ann. Sci. École Norm. Sup. (4) 19 (1986), 479-490. MR 88b:53048

[5] W. D. Dunbar Geometric orbifolds, Rev. Mat. Univ. Complu. 1 (1988) 67-99. MR 90k:22011

[6] W. Fischer and E. Koch Spanning minimal surfaces, Phil. Trans. R. Soc. Lond. A354(1996), 2105-2142. MR 97i:53009

[7] P. J. F. Gandy and J. Klinowski, Exact computation of the triply periodic G (Gyroid) minimal surface, Chem. Phys. Lett. 321 (2000), 363-371. 
[8] E. Gonzalez, U. Massari, and I. Tamanini, On the regularity of boundaries of sets minimizing perimeter with a volume constraint, Indiana Univ. Math. J. 32 (1983), 25-37. MR 84d:49043

[9] K. Grosse-Brauckmann, Gyroids of Constant Mean Curvature, Exp. Math. 6 (1997), 21-38. MR 98i:53013

[10] H. Hadwiger, Gitterperiodische Punktmengen und Isoperimetrie, Monatsh. Math. 76 (1972) 410-418. MR 48:2902

[11] T. Hahn, editor, International Tables for Crystallography, vol. A, fifth edition, Kluwer Academic Publishers, 2002.

[12] L. Hauswirth, J. Pérez, P. Romon, and A. Ros, The periodic isoperimetric problem, Trans. Amer. Math. Soc. (to appear), http://www.ugr.es/ aros/periodic.htm.

[13] S. T. Hyde, Identification of lyotropic liquid crystalline mesophases, Handbook of applied surface and colloid chemistry, edited by K. Holmberg, John Wiley \& Sons, Ltd., 2001.

[14] C. K. Johnson, M. N. Burnett, and W. D. Dunbar, Crystallographic topology and its applications, preprint.

[15] H. Karcher and K. Poltier, Construction of triply periodic minimal surfaces, Phil. Trans. R. Soc. Lond. A (1996) 354, 2077-2104. MR 97i:53008

[16] N. Korevaar, R. Kusner, and B. Solomon, The structure of complete embedded surfaces with constant mean curvature, J. Diff. Geom. 30 (1989), 465-503. MR 90g:53011

[17] E. A. Lord and A. L. Mackay, Periodic minimal surfaces of cubic symmetry, Current Science, 85 (2003), 346-362.

[18] E. Kroumova, J. M. Perez-Mato, M. I. Aroyo, S. Ivantchev, G. Madariaga, and H. Wondratschek, The Bilbao Crystallographic Server: a web site with crystallographic tools using the International Tables for Crystallography, 18th European Crystallographic Meeting, Praha, Czech Republic (1998), http://www.cryst.ehu.es.

[19] W. H. Meeks III, Lectures on Plateau's problem, Scola de Geometria Diferencial, Universidade Federal do Ceará (Brazil), 1978.

[20] F. Morgan, Regularity of isoperimetric hypersurfaces in Riemannian manifolds, Trans. Amer. Math. Soc., 355 (2003), 5041-5052.

[21] _ Regularity of area-minimizing surfaces in $3 D$ polytopes and of invariant surfaces in $\mathbb{R}^{n}$, preprint (2003).

[22] F. Morgan and D. L. Johnson, Some sharp isoperimetric theorems for Riemannian manifolds, Indiana Univ. Math. J. 49 (2000), 1017-1041. MR 2002e:53043

[23] M. O'Keeffe, J. Plévert, Y. Teshima, Y. Watanabe, and T. Ogawa, The invariant cubic rod (cylinder) packings: symmetries and coordinates, Acta Cryst. A57 (2001), 110-111. MR 2001m:52033

[24] M. O'Keeffe, J. Plévert, and T. Ogawa, Homogeneous cubic cylinder packings revisited, Acta Cryst. A58 (2001), 125-132. MR 2003c:52028

[25] M. Ritoré and A. Ros, Stable Constant Mean Curvature Tori and the Isoperimetric Problem in Three Space Forms, Comment. Math. Helvet. 67 (1992), 293-305. MR 93a:53055

[26] _ . The spaces of index one minimal surfaces and stable constant mean curvature surfaces embedded in flat three manifolds, Trans. Amer. Math. Soc. 348 (1996), 391-410. MR 96f:58038

[27] A. Ros, The isoperimetric problem, Proceedings of the Clay Mathematical Institute MSRI summer school on Minimal Surfaces (to appear), http://www.ugr.es/ aros/isoper.htm.

[28] A. H. Schoen, Infinite periodic minimal surfaces without self-intersections, NASA Technical Note No. TN D-5541 (1970).

[29] U. S. Schwarz and G. Gompper, Bicontinuous surfaces in self-assembling amphiphilic systems, Morphology of Condensed Matter: Physics and Geometry of Spatially Complex Systems, edited by K. R. Mecke and D. Stoyan, Springer Lecture Notes in Physics, Vol. 600, pp. 107-151, 2002.

[30] E. L Thomas, D. M. Anderson, C.S. Henkee, and D. Hoffman, Periodic area-minimizing surfaces in block copolymers, Nature 334 (1988), 598-602.

Departamento de Geometría y Topología, Facultad de Ciencias, Universidad de Granada, 18071 Granada, Spain

E-mail address: aros@ugr.es 